\title{
Modulation of two-color laser-induced filament terahertz emission by effec- tive length variation
}

\author{
$\underline{\text { P. A. Chizhov }}{ }^{1}$, A. A. Ushakov ${ }^{1,2}$, V. V. Bukin ${ }^{1}$, S. V. Garnov ${ }^{1}$ \\ ${ }^{1}$ A. M. Prokhorov General Physics Institute RAS, Moscow, Russia, pvch@inbox.ru \\ ${ }^{2}$ Lomonosov Moscow State University, Moscow, Russia
}

Terahertz $(\mathrm{THz})$ radiation is of great scientific interest for its applications in spectroscopy and imaging [1]. Terahertz generation in femtosecond filament in gases has the specific features as compared with other $\mathrm{THz}$ sources [2, 3]. As the gaseous media are selfhealing, one can use high-energy laser pulse focusing into small volumes without concern about material damage. Here one of the most effective ways to produce $\mathrm{THz}$ radiation is to use two-color (usually fundamental and second harmonics of fs-laser) laser pulse focusing for plasma creation [3]. However, in this case the efficiency of optical-to-terahertz conversion is highly dependent on mutual phase difference between harmonics. Moreover, for long filaments this difference varies along plasma channel. It has been demonstrated previously that this effect leads to offaxis peak in spatial $\mathrm{THz}$ distribution [4] and to possibility of waveform control by initial phase shift adjustment between harmonics [5]. In atmospheric air the distance for $\pi$ phase walk-off between fundamental and second harmonic of Ti:Sapphire laser is about $25 \mathrm{~mm}$.

In this paper, we show strong influence of phase matching between harmonics on overall $\mathrm{THz}$ power output and demonstrate that screening of a part of $\mathrm{THz}$ emission allows increasing the output power up to $20 \%$ of that for undisturbed emission. We also demonstrate the possibility of spectral modulation by means of $\pi$-retarder screens for a distinct $\mathrm{THz}$ frequency.

We use Ti:Sapphire laser system with following characteristics: $800 \mathrm{~nm}$ central wavelength, $40 \mathrm{fs}$ pulse duration, $2.8 \mathrm{~mJ}$ pulse energy, $\varnothing 12 \mathrm{~mm}\left(1 / \mathrm{e}^{2}\right.$ level), $1 \mathrm{kHz}$ repetition rate. A Glan-Taylor polarizer with a half-wave plate to control laser pulse energy is used.

A lens ( $\varnothing 25.4 \mathrm{~mm}, 1000 \mathrm{~mm}$ focal length) focuses laser radiation to create a filament in ambient air. A BBO crystal (SHG, 10x10x0.2 $\mathrm{mm}^{3}$, I-type) converting part of radiation into second harmonic is placed on the optical pump path before the plasma. After the SHG a group delay compensator plate (TP) and a dual-wave plate $(\lambda / 2 @ 800 \mathrm{~nm}+\lambda @ 400 \mathrm{~nm})$ may be placed to provide more powerful $\mathrm{THz}$ generation due to use of collinear polarizations of harmonics in twocolor pulse. The SHG and plates are mounted on a moving table to change air path of two-color pulse before the filament and so the initial phase difference between harmonics.

To collect $\mathrm{THz}$ radiation a system of two PTFElenses (first: $\varnothing 50 \mathrm{~mm}, 150 \mathrm{~mm}$ focal length; second: $\varnothing 50 \mathrm{~mm}, 100 \mathrm{~mm}$ focal length) is used. Laser pulse duration is lengthened up to $\sim 120$ fs by adding positive chirp to maximize $\mathrm{THz}$ output [6]. The length of visible plasma string is approximately $80 \mathrm{~mm}$ for maximal laser pulse energy of $2.3 \mathrm{~mJ}$.

A metallic iris diaphragm with a $\sim 1 \mathrm{~mm}$ diameter aperture centered at filament is mounted to screen $\mathrm{THz}$ radiation emitted before its position. Another moving table is utilized to control the position of the iris along the filament. Corresponding scheme is de-

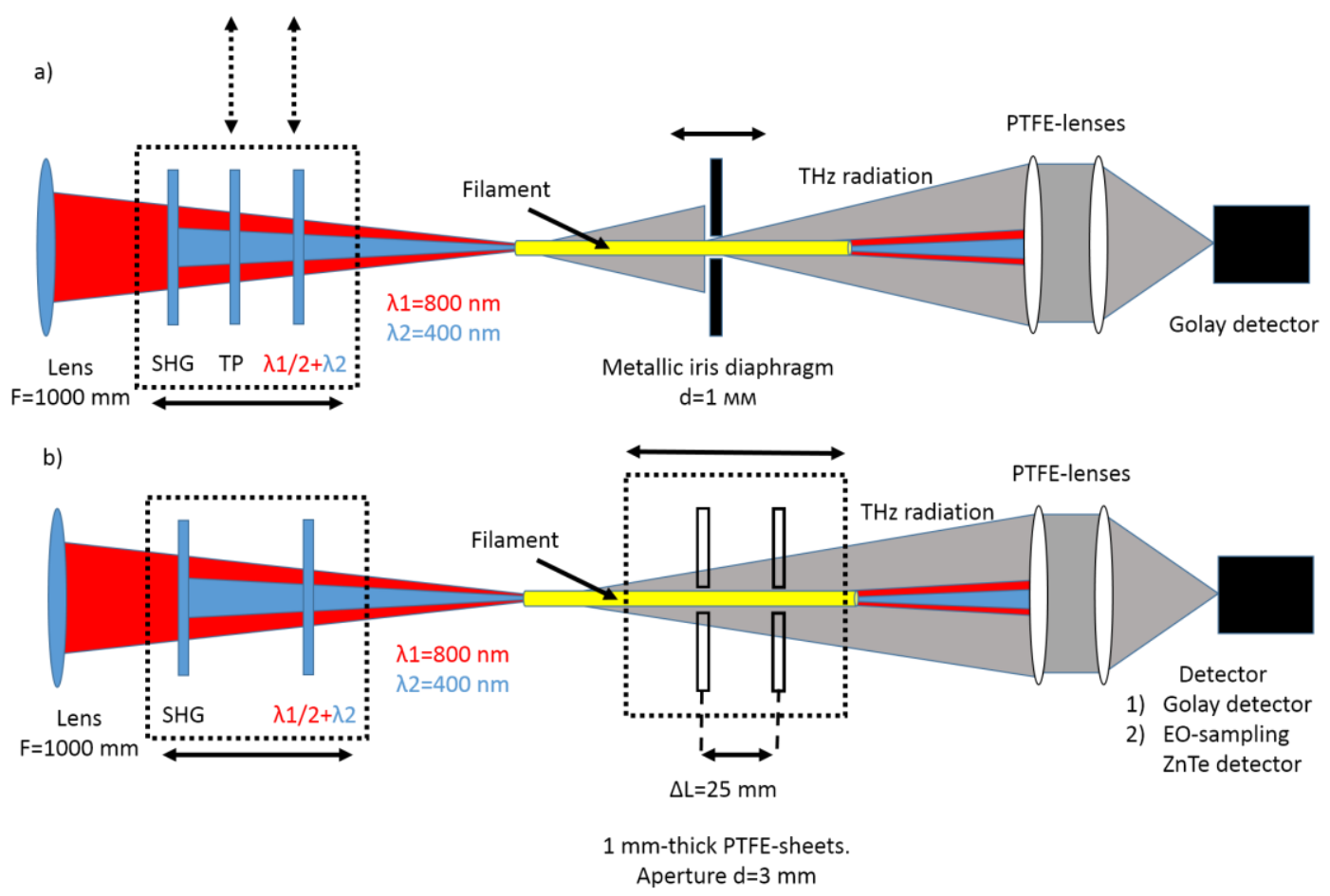

Fig. 1. Experimental schemes for: (a) screening of a part of the THz radiation with a metallic iris diaphragm; (b) spectral modulation by $\pi$-retarder PTFE-screens. 
picted on Fig.1 (a).

The second thing we want to realize is spectral and spatial modulation of the emitted $\mathrm{THz}$ radiation. The idea is to make the $\mathrm{THz}$ emission collected by PTFE-lenses from all the points along filament interfere constructively for a distinct $\mathrm{THz}$ frequency. So one need to insert additional $\pi$-retardation for the part of the $\mathrm{THz}$ radiation that has previously interfered destructively. Ideally, there should be also a non-zero on-axis value in $\mathrm{THz}$ power distribution in this case. Thus, instead of the metallic iris diaphragm we place a $1 \mathrm{~mm}$-thick ( $\pi$-retardation for $\sim 0.7 \mathrm{THz}$ ) PTFEsheet with $\sim 3 \mathrm{~mm}$ diameter aperture or a set of 2 such screens with a $25 \mathrm{~mm}$ distance between them (the distance corresponding to $\pi$ walk-off in the air). Scheme is depicted on Fig.1 (b).

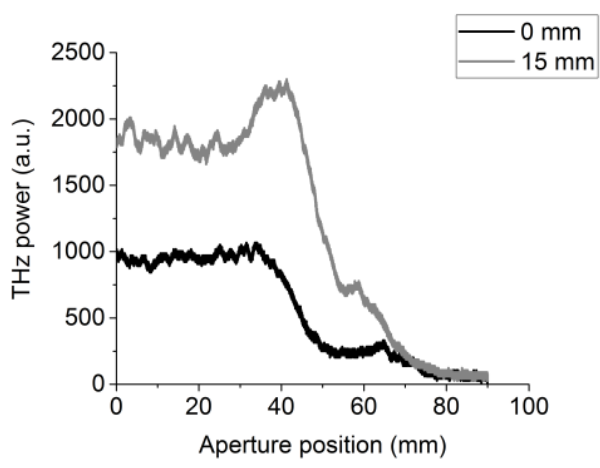

Fig. 2. Terahertz power dependence on iris diaphragm position along the filament created by $2.3 \mathrm{~mJ}$ laser-pulse for two positions of second harmonic crystal (SHG).

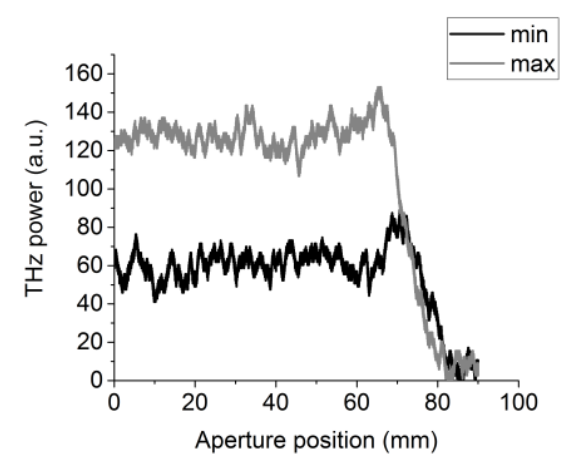

Fig. 3. Terahertz power dependence on iris diaphragm position along the filament created by $1.0 \mathrm{~mJ}$ laser pulse for two positions of second harmonic crystal (SHG).

For $\mathrm{THz}$ power measurements, a Golay cell (Tydex GC-1P) is used. For THz field measurements a standard electro-optical sampling scheme with ZnTe crystal $\left(3 \times 3 \times 1 \mathrm{~mm}^{3},<110>\right.$ cut $)$ is applied.

We measure $\mathrm{THz}$ power dependence on the iris diaphragm position along the two-color filament for different laser pulse energies from 1.0 to $2.3 \mathrm{~mJ}$ and for different initial phase difference between fundamental and second harmonics (SHG positions). We see strong influence on overall $\mathrm{THz}$ power due to constructive and destructive interference of $\mathrm{THz}$ emission from different parts of filament. For optimal initial phase and screening diaphragm position one can achieve significant increase in $\mathrm{THz}$ power. Exam- ples of such dependencies for $2.3 \mathrm{~mJ}$ laser pulse with co-polarized harmonics are given on Fig. 2.

However, for short filament (low laser pulse energy) the effect of phase walk-off along plasma channel is sufficiently decreasing. See dependencies for $1.0 \mathrm{~mJ}$ laser pulse with co-polarized harmonics on Fig. 3.

For the experiment on spectral modulation, we collected waveforms of $\mathrm{THz}$ pulses and $\mathrm{THz}$ power value for different positions of $\pi$-retarder screen/set of screens. Thereafter waveforms are processed with fast Fourier transform to get spectra of $\mathrm{THz}$ pulses. Here one can see significant narrowing of spectrum for some position of the screens with peak amplitude on $0.7 \mathrm{THz}$ frequency, that corresponds to the thickness of the PTFE-sheets used. There is also a bright peak on the doubled frequency $-1.4 \mathrm{THz}$. However, spectral change is accompanied with a dramatic drop in emitted $\mathrm{THz}$ power, so that even the amplitude on peak frequency is lower than that in spectrum with no screens placed. On Fig. 4 are given spectra of $\mathrm{THz}$ pulses for different positions of the set of two PTFEscreens: $0 \mathrm{~mm}$ position corresponds to undisturbed emission (screens placed before the whole filament), $40 \mathrm{~mm}$ position corresponds to significant influence of the $\pi$-retarders on emitted $\mathrm{THz}$ radiation.

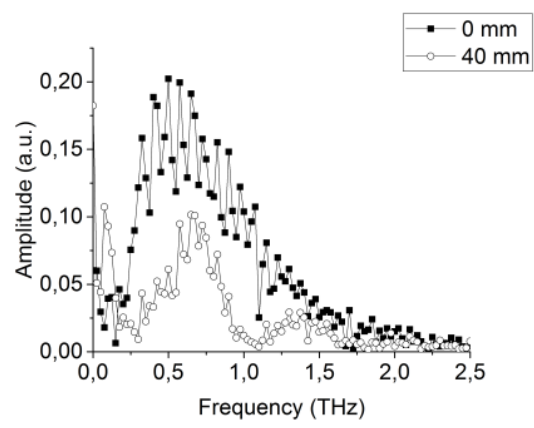

Fig. 4. Terahertz pulse spectra for two PTFE-screens positions.

\section{References}

1. Zhang, X.-C., Shkurinov, A., Zhang, Y. Extreme terahertz science // Nature Photonics. 2017. V. 11, No. 1. P. 16-18.

2. Hamster, H., Sullivan, A., Gordon, S., White, W., Falcone, R. W. Subpicosecond, Electromagnetic Pulses from Intence Laser-Plasma Interaction // Phys. Rev. Lett. 1993. V. 71, No. 17. P. 2725-2728.

3. Cook, D. J., Hochstrasser R. M. Intense terahertz pulses by four-wave rectification in air // Opt. Lett. 2000. V. 25, No. 18. P. 1210-1212

4. You, Y. S., Oh, T. I., Kim, K. Y. Off-Axis PhaseMatched Terahertz Emission from Two-Color LaserInduced Plasma Filaments // Phys. Rev. Lett. 2012. V. 109, No. 18. P. 183902.

5. Zhang, Z., Chen, Y., Chen, M., Zhang, Z., Yu, J., Sheng, Z., Zhang, J. Controllable Terahertz Radiation from a Linear-Dipole Array Formed by a Two-Color Laser Filament in Air // Phys. Rev. Lett. 2016. V. 117, No. 24. P. 243901.

6. Wang, T.-J., Chen, Y., Marceau, C., Théberge, F., Châteauneuf, M., Dubois, J., Chin, S. L. High energy terahertz emission from two-color laser-induced filamentation in air with pump pulse duration control // Appl. Phys. Lett. 2009. V. 95, No. 18. P. 131108. 